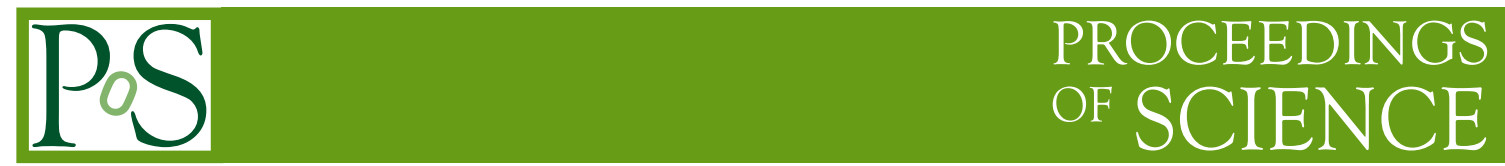

\title{
Heavy flavour production at LHCb
}

\section{Sergey Barsuk* for the LHCb Collaboration}

LAL (IN2P3/CNRS and Paris-Sud University)

E-mail: sergey.barsukecern.ch

The present write-up reports recent $\mathrm{LHCb}$ results on production of quarkonium and open flavour states, as well as selected results on associated production, central exclusive production and production in heavy ion collisions.

16th International Conference on B-Physics at Frontier Machines

2-6 May 2016

Marseille, France

${ }^{*}$ Speaker. 


\section{Introduction}

$\mathrm{LHCb}[1,2]$ is an experiment mainly dedicated to precision studies of $C P$ violation and decays of heavy-flavoured hadrons with the aim of constraining relevant Standard Model (SM) parameters and search for non-explainable phenomena within the SM. In addition, the experiment is well suited to perform unique measurements of heavy flavour production, which provide powerful tests of QCD. Being hosted by the LHC, which is presently the richest source of $c$ and $b$ hadrons, LHCb is designed to fully exploit this potential. The $\mathrm{LHCb}$ detector is a single-arm forward spectrometer covering the acceptance of $10-250$ (300) mrad in vertical (horizontal) direction. Forward peaked heavy quark production at the LHC motivated the chosen detector geometry. In addition, there is strong correlation between the quark and anti-quark flight directions in the pair production. Thus while covering about $4 \%$ of the solid angle, LHCb measures about $40 \%$ of heavy quark production cross-section. The LHCb acceptance is complementary to that of the ATLAS and CMS detectors, and allows complementary production cross-section measurements including rapidity overlap. Production studies addressed in the present write-up rely on precision track and vertex reconstruction by a silicon vertex locator [3,4], powerful identification capabilities of muons and charged hadrons by the muon detector [5, 6] and ring imaging Cherenkov detectors [7, 8], respectively, and a flexible trigger [9].

\section{Quarkonium production}

The theoretical description of heavy quarkonium production involves both perturbative and non-perturbative QCD regimes to describe short-distance $Q \bar{Q}$ pair production and hadronisation, respectively. New LHCb results on the production of $J^{P C}=1^{--}$states, $J / \psi$ at $\sqrt{s}=13 \mathrm{TeV}$ and $\Upsilon(n S)$ at $\sqrt{s}=7$ and $8 \mathrm{TeV}$, and $0^{-+}$state, $\eta_{c}(1 S)$ at $\sqrt{s}=7$ and $8 \mathrm{TeV}$, are discussed below.

Double differential $J / \psi$ production cross-section is measured in bins of transverse momentum $p_{T}$ and rapidity $y$. Prompt and $b$-decay components are extracted from the fit to pseudo-lifetime distribution. Using an integrated luminosity of $\int L d t \sim 3 \mathrm{pb}^{-1}$, LHCb measures the $J / \psi$ production cross-section at $\sqrt{s}=13 \mathrm{TeV}$ integrated over acceptance to be [10]

$$
\begin{aligned}
\sigma\left(\operatorname{prompt} J / \psi, p_{T}<14 \mathrm{GeV} / \mathrm{c}, 2.0<y<4.5\right) & =15.30 \pm 0.03 \pm 0.86 \mu b, \\
\sigma\left(J / \psi-\text { from }-\mathrm{b}, p_{T}<14 \mathrm{GeV} / \mathrm{c}, 2.0<y<4.5\right) & =2.34 \pm 0.01 \pm 0.13 \mu b .
\end{aligned}
$$

The latter cross-section is translated to the $b \bar{b}$ production cross-section integrated over $4 \pi$ of $\sigma(p p \rightarrow b \bar{b} X)=515 \pm 2 \pm 53 \mu \mathrm{b}$. Using this result together with previous measurements at $\sqrt{s}=2.76,7$, and $8 \mathrm{TeV}[11,12,13]$ the cross-section dependence on the center-of-mass energy is found to be in agreement with the FONLL prediction from Ref. [14].

Both experimental and model-related systematic uncertainties partly cancel in ratios of production cross-sections at different $\sqrt{s}$. The ratio of the $J / \psi$ differential production cross-sections at $\sqrt{s}=13$ and $8 \mathrm{TeV}$ is shown in Fig. 1 as a function of the transverse momentum for prompt production and of the rapidity for production from $b$ decays. Some tension is observed between the measurements and NRQCD [15] and FONLL [16] calculations.

Double differential production cross-sections of the $\Upsilon(n S)$ states at $\sqrt{s}=7$ and $8 \mathrm{TeV}$ are measured using an integrated luminosity of $\int L d t \sim 1$ and $2 \mathrm{fb}^{-1}$, respectively, in the kinematic 

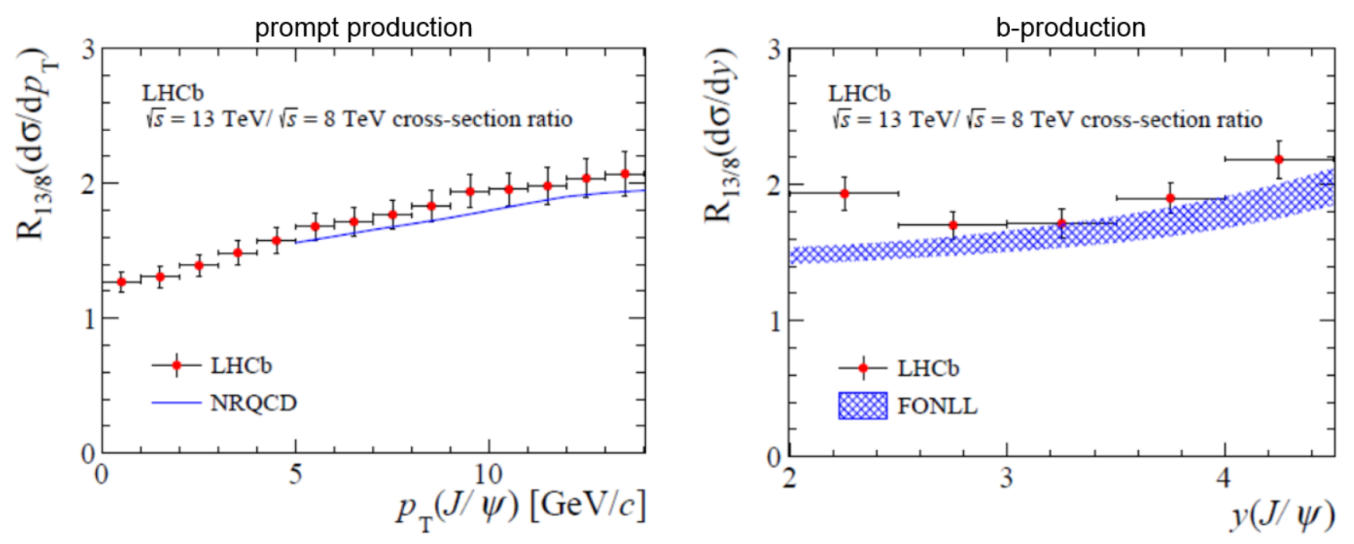

Figure 1: Ratio of the $J / \psi$ differential production cross-sections at $\sqrt{s}=13$ and $8 \mathrm{TeV}$. Transverse momentum dependence for prompt production (left) and rapidity dependence for production in $b$-decays (right). Predictions obtained using NRQCD [15] and FONLL [16] calculations are also shown.

\begin{tabular}{l|r|r} 
& $\sqrt{s}=7 \mathrm{TeV}$ & $\sqrt{s}=8 \mathrm{TeV}$ \\
\hline$\sigma^{\Upsilon(1 S) \rightarrow \mu^{+} \mu^{-}}$ & $2510 \pm 3 \pm 80$ & $3280 \pm 3 \pm 100$ \\
$\sigma^{\Upsilon(2 S) \rightarrow \mu^{+} \mu^{-}}$ & $635 \pm 2 \pm 20$ & $837 \pm 2 \pm 25$ \\
$\sigma^{\Upsilon(3 S) \rightarrow \mu^{+} \mu^{-}}$ & $313 \pm 2 \pm 10$ & $393 \pm 1 \pm 12$
\end{tabular}

Table 1: Cross sections for $\Upsilon(n S)$ production (in $\mathrm{pb}$ ).
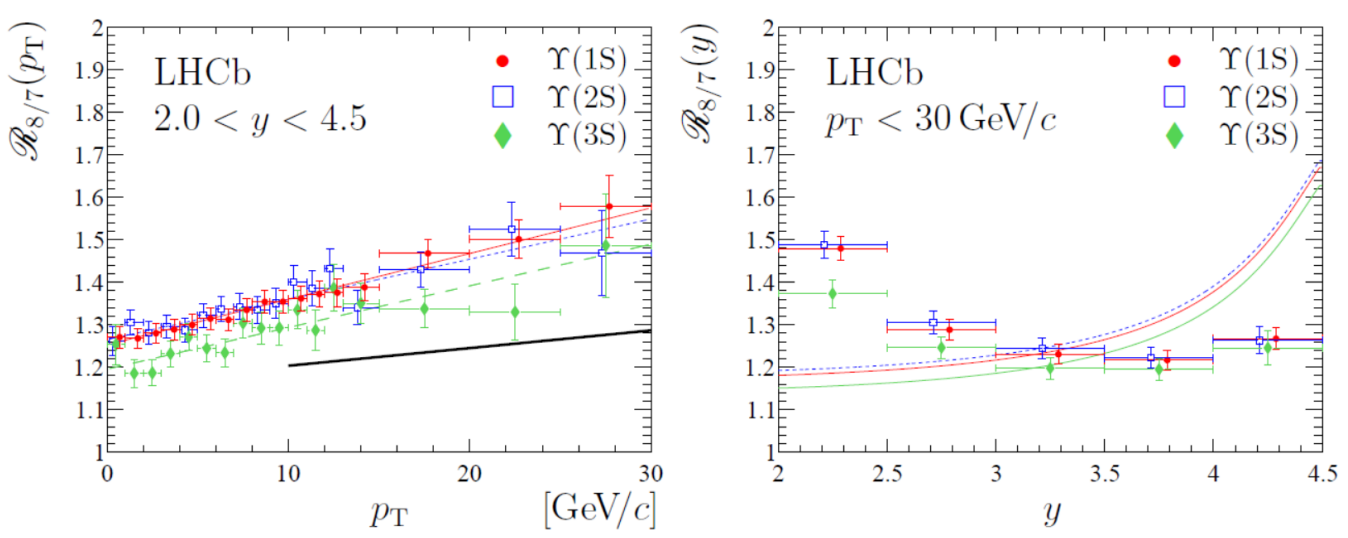

Figure 2: Ratios of the $\Upsilon(n S)$ production cross-sections at $\sqrt{s}=8$ and $7 \mathrm{TeV}$. Transverse momentum (left) and rapidity (right) dependences. Prediction curves obtained using the NRQCD [18] and CO model [19, 20, 21] calculations are shown on the left and right plots, respectively.

regime $p_{T}<30 \mathrm{GeV} / \mathrm{c}^{2}, 2.0<y<4.5$ [17]. Table 1 summarizes the results. Similarly to the $J / \psi$ case, the $\Upsilon(n S)$ production cross-section ratios $R_{8 / 7}$ are compared to the NRQCD [18] and Colour Octet (CO) model calculations in Fig. 2 [19, 20, 21]. Observed increase in the production crosssections between $\sqrt{s}=7$ and $8 \mathrm{TeV}$ significantly exceeds theoretical expectations, and exceeds that measured in the $J / \psi$ production cross-section. The observed trend of $R_{8 / 7}$ is not described with pure $\mathrm{CO}$ mechanism.

Quarkonium reconstruction through experimentally clean di-muon final states is only possible for $J^{P C}=1^{--}$states. In addition, the $\chi$ states can be reconstructed via radiative decays to the $J^{P C}=$ 

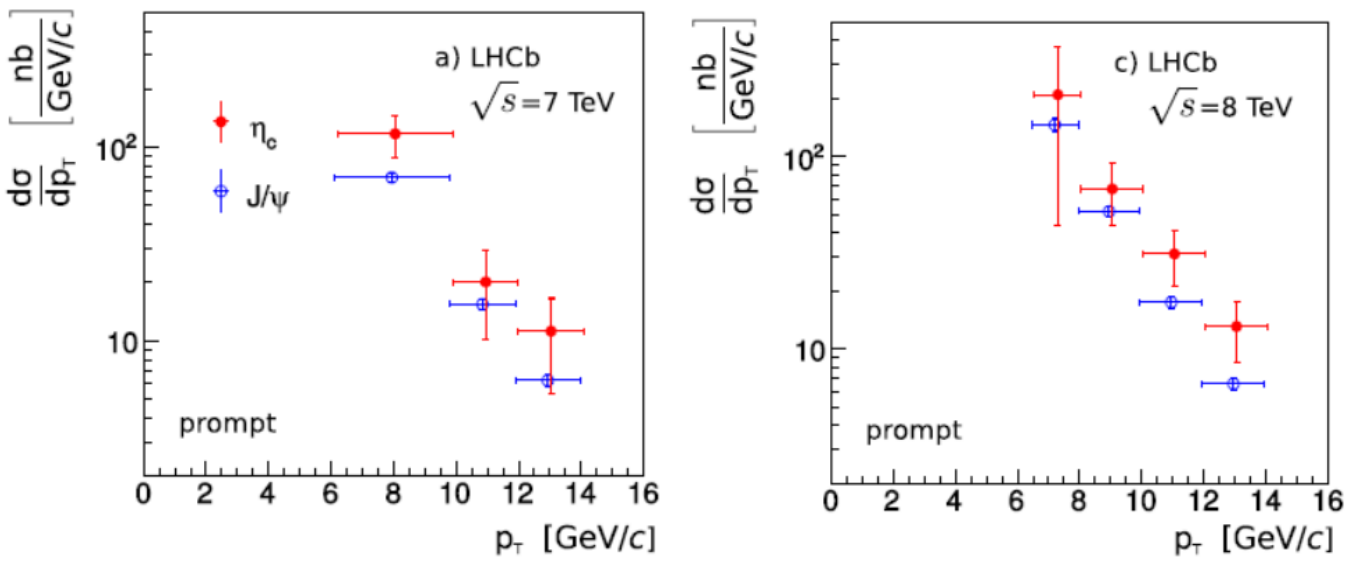

Figure 3: Differential prompt production cross-section of the $\eta_{c}(1 S)$ and $J / \psi$ states at $\sqrt{s}=7 \mathrm{TeV}$ (left) and $8 \mathrm{TeV}$ (right).

$1^{--}$state and a low-energy photon. Using decays to hadronic final states LHCb has access to the production of other quarkonium states [22]. With an integrated luminosity of $\int L d t \sim 1$ and $2 \mathrm{fb}^{-1}$ accumulated at $\sqrt{s}=7$ and $8 \mathrm{TeV}$, respectively, LHCb measures for the first time the production of the $\eta_{c}(1 S)$ state using the $\eta_{c}(1 S) \rightarrow p \bar{p}$ channel [23]. Production relative to that of the $J / \psi$ is measured with the absolute production cross-section determined using the $J / \psi$ production crosssection from Ref. $[12,13]$. Prompt $\eta_{c}(1 S)$ production and production in $b$-decays are distinguished using separation between the $p p$ interaction and the charmonium decay vertices. Production in $b$ decays is measured to be $B R\left(b \rightarrow \eta_{c}(1 S) X\right)=\left(4.88 \pm 0.64 \pm 0.29 \pm 0.67_{B R}\right) \times 10^{-3}$, where the last uncertainty is due to the uncertainty in the branching fractions of the $b \rightarrow J / \psi X$ and $\eta_{c}(1 S) \rightarrow p \bar{p}$ decays. Prompt $\eta_{c}(1 S)$ production cross-section in the $\mathrm{LHCb}$ kinematic regime is measured to be

$$
\begin{aligned}
& \sigma\left(\eta_{c}(1 S), \sqrt{s}=7 \mathrm{TeV}\right)=0.52 \pm 0.09 \pm 0.08 \pm 0.06 \mu b \\
& \sigma\left(\eta_{c}(1 S), \sqrt{s}=8 \mathrm{TeV}\right)=0.59 \pm 0.11 \pm 0.09 \pm 0.08 \mu b
\end{aligned}
$$

where the last uncertainty is due to uncertainties in the branching fractions of the $\eta_{c}(1 S)$ and $J / \psi$ meson decays to $p \bar{p}$. Differential prompt production cross-section is shown in Fig. 3 for $\sqrt{s}=7$ and $8 \mathrm{TeV}$ and compared to that of $J / \psi$. Different $p_{T}$ spectra for $\eta_{c}(1 S)$ and $J / \psi$ suggested in Refs. $[24,25,26]$ are not observed by LHCb. Using the relations between matrix elements and Long Distance Matrix Elements calculated in Refs. [27, 28, 29, 30] from the $J / \psi$ production measurements, the authors of Ref. [31] confront the theory predictions to the LHCb results. While being described by the Colour Singlet (CS) NLO contribution, the measured cross-section is below the $\mathrm{CO}$ expectations.

\section{Production of open flavours}

Measurements of open charm production provide powerful tests of perturbative QCD. In addition, they bring important information on the rate of high-energy neutrinos from charm-hadron decays produced in cosmic ray interactions with atmospheric nuclei. LHCb measures prompt production cross-sections of charmed mesons with the very first data of the 2015 run, corresponding 

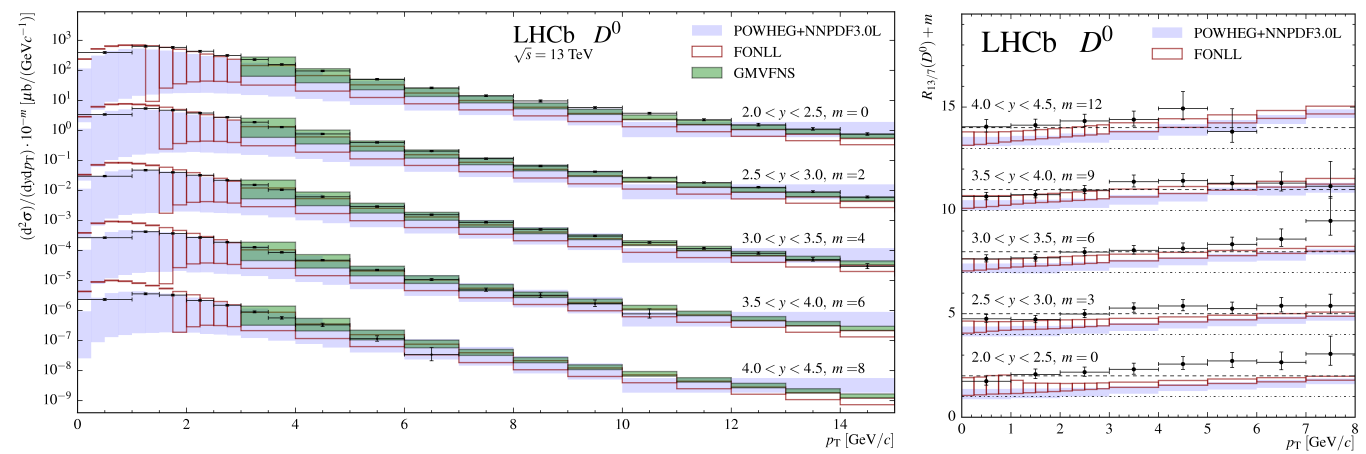

Figure 4: Differential $D^{0}$ prompt production cross-section with the predictions from Refs. [33, 16, 34] overlaid as grey, empty, and green rectangles, respectively (left). Cross-section ratios for $\sqrt{s}=13$ to $7 \mathrm{TeV}$ and predictions from Refs. [33] and [16] are overlaid as grey and empty rectangles, respectively (right).

to an integrated luminosity $\int L d t \sim 5 \mathrm{pb}^{-1}$ at $\sqrt{s}=13 \mathrm{TeV}$ [32]. In order to select prompt charmed mesons, fits to the $\ln \chi^{2}$ of the impact parameter with respect to the $p p$ interaction vertex are performed. Integrated inclusive cross-sections in the $1<p_{T}<8 \mathrm{GeV} / \mathrm{c}^{2}, 2.0<y<4.5$ kinematic regime are found to be

$$
\begin{gathered}
\sigma\left(p p \rightarrow D^{0} X\right)=2470 \pm 3 \pm 130 \mu b, \\
\sigma\left(p p \rightarrow D^{+} X\right)=1000 \pm 3 \pm 110 \mu b, \\
\sigma\left(p p \rightarrow D_{s}^{+} X\right)=460 \pm 13 \pm 100 \mu b \\
\sigma\left(p p \rightarrow D^{*+} X\right)=880 \pm 5 \pm 140 \mu b .
\end{gathered}
$$

Differential production cross-sections are determined in the $1<p_{T}<15 \mathrm{GeV} / \mathrm{c}^{2}, 2.0<y<4.5$ kinematic regime. The measured $D^{0}$ differential production cross-section and relative production cross-section between $\sqrt{s}=13$ and $7 \mathrm{TeV}, R_{13 / 7}$, are confronted to the POWHEG+NNPDF3.0L [33], FONLL [16], and GMVFNS [34] model predictions in Fig. 4. The measured cross-sections are higher than theory expectations but within uncertainties. Using the Blue method [35], the $c \bar{c}$ production cross-section in the $0<p_{T}<8 \mathrm{GeV} / \mathrm{c}^{2}, 2.0<y<4.5$ kinematic regime is determined from meson measurements, to be

$$
\sigma(p p \rightarrow c \bar{c} X)=2840 \pm 3 \pm 170 \pm 150 \mu b .
$$

This value agrees with the absolute cross-section predictions from Refs. [33, 16] within uncertainties, and differs from the reduced-uncertainty scaled cross-section prediction from Ref. [33] by more than two standard deviations.

Kinematic dependence of the production of $b$-baryons relative to that of $B$-mesons probes differences in the $b$ quark hadronisation processes. LHCb measures $\Lambda_{b}$ production at $\sqrt{s}=7$ and $8 \mathrm{TeV}$ with $\int L d t \sim 3 \mathrm{fb}^{-1}$, using the $\Lambda_{b} \rightarrow J / \psi K \pi$ decay mode [36]. The differential cross-section ratio between $\sqrt{s}=7$ and $8 \mathrm{TeV}$ is generally in agreement with the FONLL predictions [14, 37, 38], while some tension is observed at low $y$ values. Fig. 5, left shows the $\Lambda_{b}$ and $B^{0}$ production ratio, $R_{\Lambda_{b} / \bar{B}^{0}}$, as a function of $p_{T}$, confirming the $p_{T}$ dependence of the fragmentation function. Nonzero slope in the $\Lambda_{b}-\bar{\Lambda}_{b}$ asymmetry at the level of more than four standard deviations provides an evidence for string drag effects [39]. 

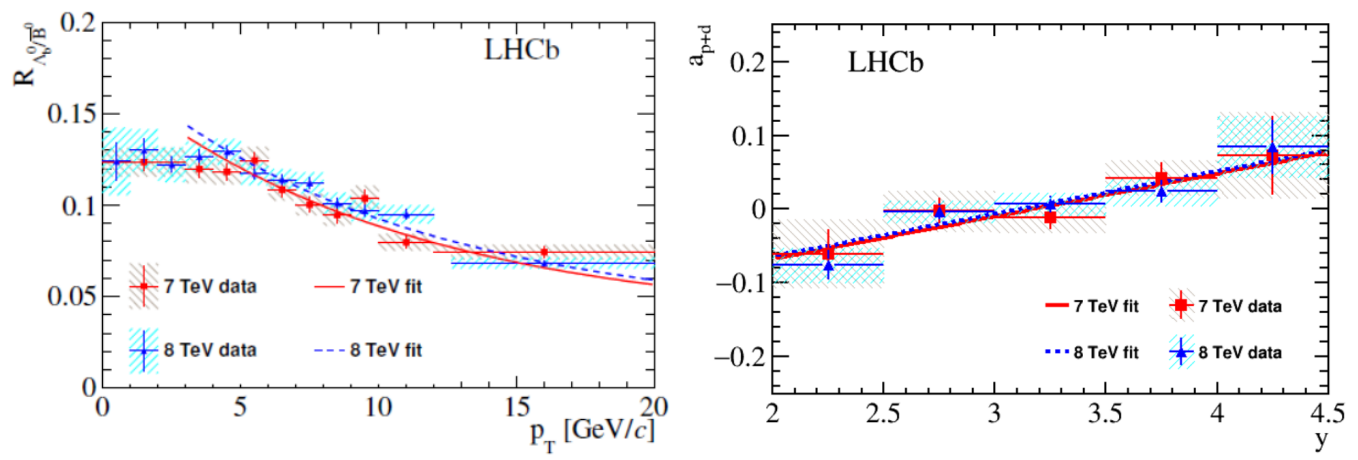

Figure 5: The $\Lambda_{b}$ and $B^{0}$ production ratio, $R_{\Lambda_{b} / \bar{B}^{0}}$ as a function of $p_{T}$ (left) and the $\Lambda_{b}-\bar{\Lambda}_{b}$ asymmetry as a function of rapidity $y$ (right).
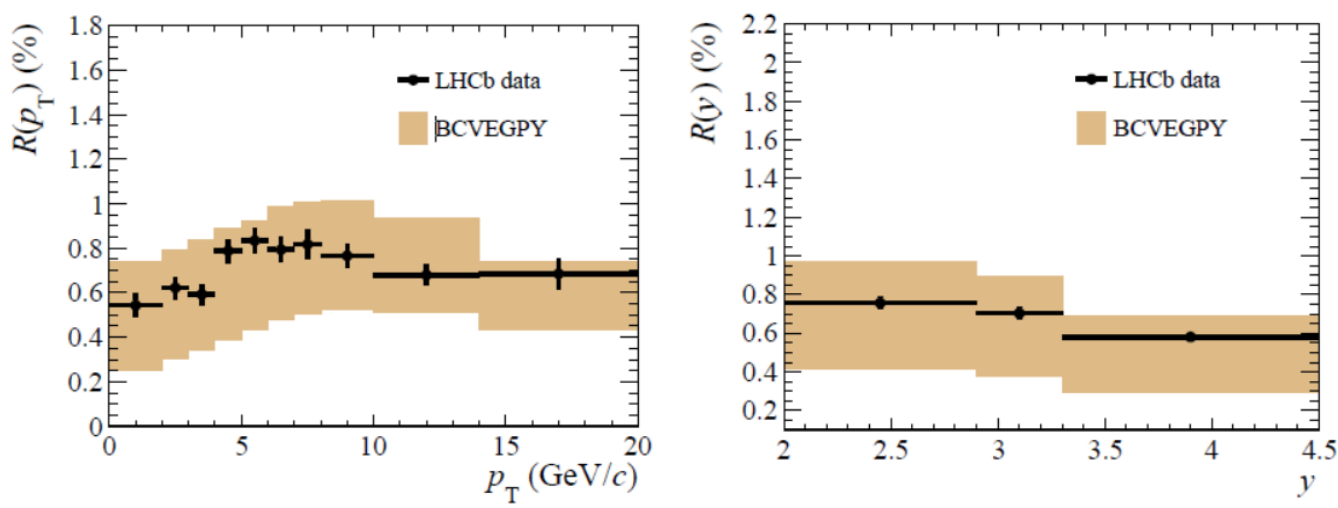

Figure 6: Production ratio $R$ as a function of $p_{T}$ (left) and $y$ (right).

Production measurement of the $B_{c}^{+}$meson probes simultaneous production of $b$ - and $c$-quark pairs via gluon-gluon fusion. Using integrated luminosity of $\int L d t \sim 2 \mathrm{fb}^{-1}$ accumulated at $\sqrt{s}=$ $8 \mathrm{TeV}$, LHCb reconstructs a sample of $3100 B_{c}^{+} \rightarrow J / \psi \pi^{+}$decays [45]. Relative integrated $B_{c}^{+}$and $B^{+}$production is measured as

$$
R \equiv \frac{\sigma\left(B_{c}^{+}\right) \times B R\left(B_{c}^{+} \rightarrow J / \psi \pi^{+}\right)}{\sigma\left(B^{+}\right) \times B R\left(B^{+} \rightarrow J / \psi K^{+}\right)}=(0.683 \pm 0.018 \pm 0.009) \% .
$$

The production ratio dependence on transverse momentum and rapidity $R=R\left(p_{T}, y\right)$ is in agreement with BCVEGPY order $\alpha_{s}^{4}$ predictions [41, 42] (Fig. 6).

\section{Associated production}

Studies of associated production of two heavy flavour hadrons aims at distinguishing between Double Parton Scattering (DPS) with two independent hard scatters that are assumed to factorize and Single Parton Scattering (SPS) with dominant contribution of gluon splitting. While the LHCb measurement of double $J / \psi$ production is in agreement with both SPS and DPS expectations [43]. the double charm production cross-section involving open charm exceeds the SPS predictions [44]. The associated $(b \bar{b})(c \bar{c})$ production accessed via the production of $B_{c}^{+}$agrees with the SPS calculations [45]. Another probe of the associated $(b \bar{b})(c \bar{c})$ production is obtained by studying simultaneous production of $\Upsilon(n S)$ and open charm. The ratio of the associated production of $\Upsilon(n S)$ and open 

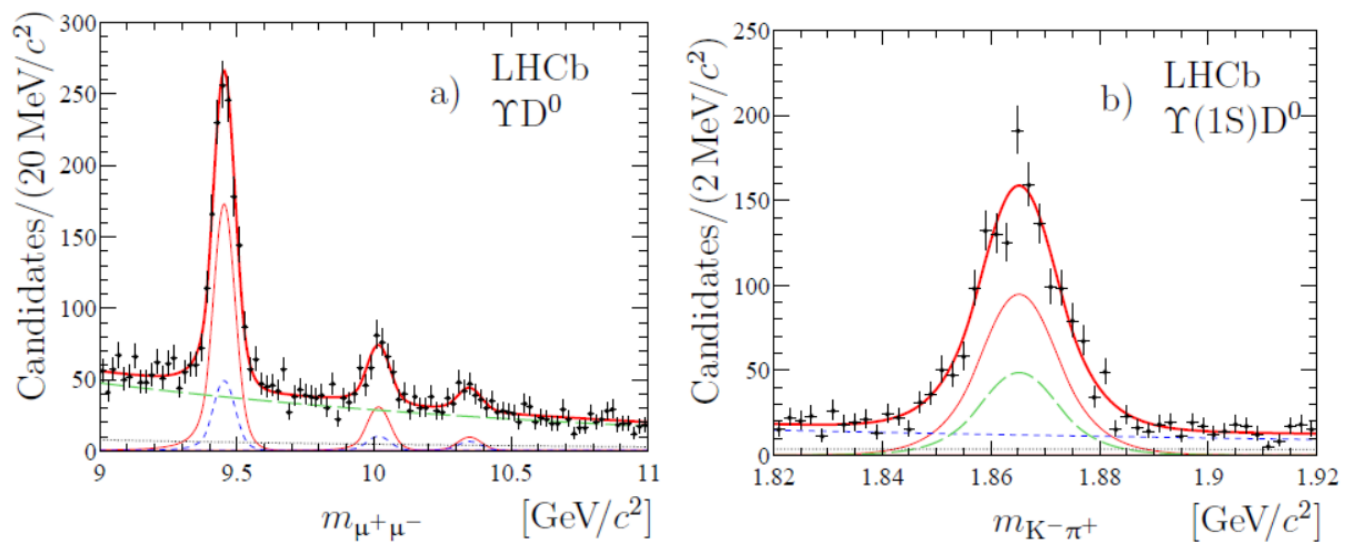

Figure 7: Associated $\Upsilon D^{0}$ production: projections from two-dimensional extended unbinned maximum likelihood fit. The total fit function (solid thick red curve), the $\Upsilon D^{0}$ signal components (solid thin red curves), components describing $\Upsilon$ signals and combinatorial background in $K^{-} \pi^{+}$mass (short-dashed blue curves), the component modelling the true $D^{0}$ signal and combinatorial background in $\mu^{+} \mu^{-}$mass (longdashed green curve) and the component describing combinatorial background (thin dotted black line) are shown.

charm to the $\Upsilon(n S)$ production in the LHCb acceptance is predicted as $R_{D P S} \equiv \frac{\sigma(\Upsilon c \bar{c})}{\sigma(\Upsilon)}=\frac{\sigma(c \bar{c})}{\sigma_{e f f}} \sim 10 \%$ if proceeded via DPS, where $\sigma_{\text {eff }}$ is the effective cross-section, and as $R_{S P S} \equiv \frac{\sigma(\Upsilon c \bar{c})}{\sigma(\Upsilon)}=(0.2-0.6) \%$ if proceeded via SPS [46]. LHCb studies associated $(\Upsilon c \bar{c})$ production using an integrated luminosity of $\int L d t \sim 3 \mathrm{fb}^{-1}$ accumulated at $\sqrt{s}=7$ and $8 \mathrm{TeV}$ [47]. The signal yield is determined using a $2 \mathrm{D}$ extended unbinned maximum likelihood fit, while the contribution from the pile-up is determined from data. Fit projections are illustrated in Fig. 7, where contributions from the $\Upsilon D$, $\left(\mu^{+} \mu^{-}\right) D, \Upsilon(K \pi)$ and $\left(\mu^{+} \mu^{-}\right)(K \pi)$ signals are shown separately. LHCb reports an observation of the $\left(\Upsilon(1 S) D^{0}\right),\left(\Upsilon(2 S) D^{0}\right),\left(\Upsilon(1 S) D^{+}\right),\left(\Upsilon(2 S) D^{+}\right)$, and $\left(\Upsilon(1 S) D_{s}^{+}\right)$associated productions and measures cross-sections for $\left(\Upsilon(1 S) D^{0}\right)$ and $\left(\Upsilon(1 S) D^{+}\right)$to be

$$
\begin{aligned}
& \sigma_{\sqrt{s}=7}^{\Upsilon(1 S) D^{0}} \times B R\left(\Upsilon(1 S) \rightarrow \mu^{+} \mu^{-}\right)=155 \pm 21 \pm 7 p b, \\
& \sigma_{\sqrt{s}=7}^{\Upsilon(1 S) D^{+}} \times B R\left(\Upsilon(1 S) \rightarrow \mu^{+} \mu^{-}\right)=82 \pm 19 \pm 5 p b, \\
& \sigma_{\sqrt{s}=8}^{\Upsilon(1 S) D^{0}} \times B R\left(\Upsilon(1 S) \rightarrow \mu^{+} \mu^{-}\right)=250 \pm 28 \pm 11 p b, \\
& \sigma_{\sqrt{s}=8}^{\Upsilon(1 S) D^{+}} \times B R\left(\Upsilon(1 S) \rightarrow \mu^{+} \mu^{-}\right)=80 \pm 16 \pm 5 p b,
\end{aligned}
$$

in the $2.0<y^{\Upsilon}<4.5, p_{T}^{\Upsilon}<15 \mathrm{GeV} / \mathrm{c}, 2.0<y^{D}<4.5,1<p_{T}^{D}<20 \mathrm{GeV} / \mathrm{c}$ fiducial region. Measured cross-sections are found to be in agreement with DPS, while significantly exceeding the SPS predictions. Differential kinematic distributions also suggest DPS as the main production mechanism. The value of the effective cross-section is determined from the cross-sections of the $\left(\Upsilon(1 S) D^{0}\right)$ and $\left(\Upsilon(1 S) D^{+}\right)$associated production to be $\sigma_{e f f}=18.0 \pm 1.3 \pm 1.2 \mathrm{mb}$, in agreement with previous measurements.

\section{Central Exclusive production}

Central Exclusive Production (CEP) provides a tool for precision QCD tests with clean theo- 

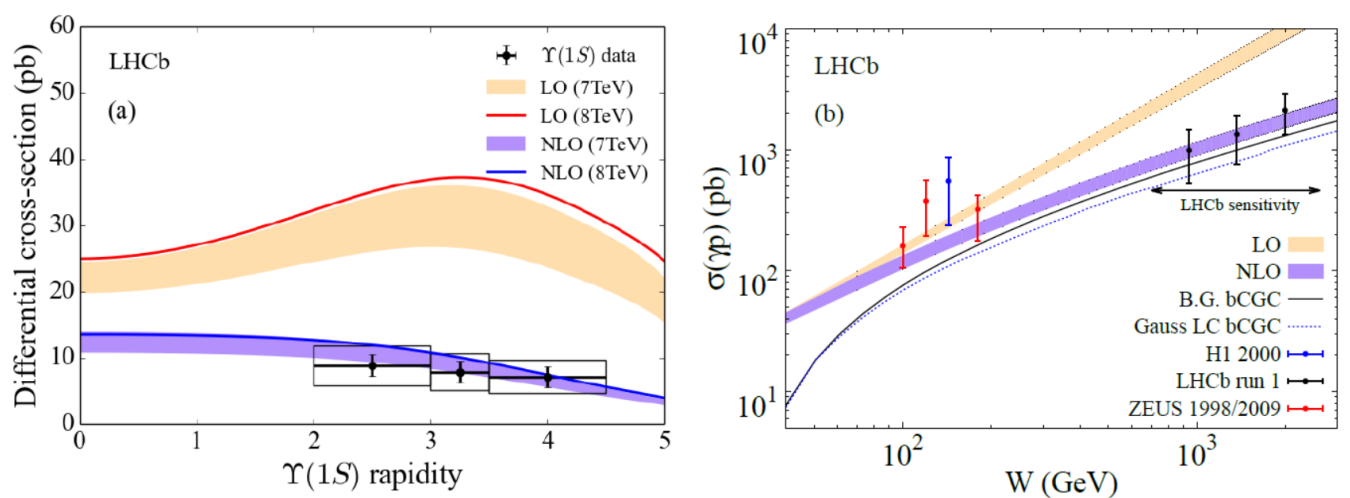

Figure 8: The exclusive $\Upsilon(1 S)$ cross-section in bins of rapidity compared to LO and NLO predictions (left) and the photon-proton cross-sections extracted from the LHCb results and the entire $W$-region in which these LHCb measurements are sensitive (right). Results from HERA [50, 51, 52], theory predictions from the LO and NLO fits to exclusive $J / \psi$ production data [49], and predictions from Ref. [53] using different models for the $\Upsilon(1 S)$ wave function (bCGC), are overlaid. The filled bands indicate the theoretical uncertainties on the $7 \mathrm{TeV}$ prediction and the solid lines indicate the central values of the predictions for $8 \mathrm{TeV}$.

retical interpretation. Cross-section measurements in the LHCb coverage provide sensitivity down to $x$ values of about $1.5 \times 10^{-5}$. With an integrated luminosity of $\int L d t \sim 3 \mathrm{fb}^{-1}$ accumulated at $\sqrt{s}=7$ and $8 \mathrm{TeV}$ and a dedicated CEP trigger, LHCb measures CEP of $\Upsilon(n S)$ [48]. Exclusive selection of di-muon pairs is quantified with the fit to the $p_{T}$ spectrum. Feed-down contributions from the $\chi_{b}(m P) \rightarrow \Upsilon(n S) \gamma$ decays are estimated using data and simulation. Cross-sections at $2.0<y<4.5$ are measured to be

$$
\begin{aligned}
& \sigma(p p \rightarrow p \Upsilon(1 S) p)=9.0 \pm 2.1 \pm 1.7 p b, \\
& \sigma(p p \rightarrow p \Upsilon(2 S) p)=1.3 \pm 0.8 \pm 0.3 p b, \\
& \sigma(p p \rightarrow p \Upsilon(3 S) p)<3.4 p b @ 95 \% C L .
\end{aligned}
$$

The comparison of measured cross-sections to theory predictions [49] (Fig. 8, left) highlights the importance of the NLO calculations. LHCb probes a previously unexplored kinematic region in the photon-proton center-of-mass energy $W$ [53] (Fig. 8, right). Improved exclusivity determination and trigger efficiency for CEP at LHCb are expected during LHC Run II with a new scintillator detector at high rapidity, increasing the rapidity gap by 6 units.

\section{Heavy flavour production in heavy ion collisions}

Suppression of heavy quarkonia production in heavy ion collisions provides a signature of Quark-Gluon Plasma (QGP) formation. However a contribution from Cold Nuclear Matter (CNM) effects should be subtracted. The expected CNM contribution could be studied in proton-ion collisions. LHCb studies the production of $J / \psi$ [54], $\psi(2 S)$ [55] and $\Upsilon(n S)$ states [56] in $p P b$ collisions at $\sqrt{s_{N N}}=5 \mathrm{TeV}$. Double differential cross-sections of the prompt $\psi(2 S)$ production and production from $b$-hadron decays are measured in the $0<p_{T}<14 \mathrm{GeV} / \mathrm{c},-5<y<-2.5$ and $1.5<y<4$ kinematic regime. Along with the $J / \psi$ production measurements, the $b \bar{b}$ production cross-section in $p P b$ collisions can be determined. Measurements of the nuclear modification factor $R_{p P b}$ and 

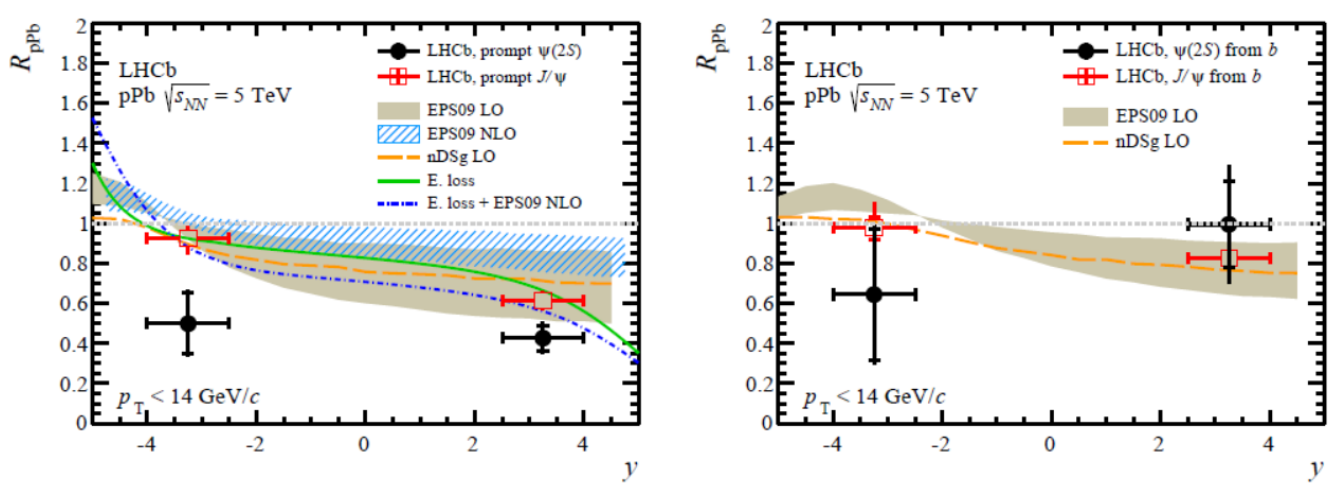

Figure 9: Nuclear modification factor $R_{p P b}$ as a function of y for prompt $\psi(2 S)$ with theoretical predictions from Refs. [57, 58] shown as yellow dashed line and brown band, from Ref. [59] (blue band) and from Ref. [60] shown as green solid and blue dash-dotted lines (left), and $\psi(2 S)$ from $b$-decays with theoretical prediction from Ref. [58] (right).

of the forward-backward ratio $R_{F B}$ show prompt $\psi(2 S)$ production to be significantly more suppressed than that of $J / \psi$ mesons in the backward region. These measurements are compared to the theoretical predictions $[57,58,59,60]$ in Fig. 9. The production rate of the $\psi(2 S)$ state is not well described by theoretical predictions based on shadowing and energy loss mechanisms.

\section{Summary}

LHCb has delivered new results on heavy flavour production, including production of quarkonium and open flavour states, associated production, central exclusive production, and heavy flavour production in heavy ion collisions. using Run I and Run II data. These measurements enable precision tests of QCD to be made. A further increase of data sample sizes will allow for even improved sensitivities.

\section{References}

[1] A. A. Alves, Jr. et al., LHCb Collab., JINST 3 (2008) S08005.

[2] R. Aaij et al., LHCb Collab., Int. J. Mod. Phys. A30, 7 (2015) 1530022.

[3] R. Aaij et al., JINST 9 (2014) 09007.

[4] A. Affolder et al., JINST 8 (2013) P08002.

[5] A. A. Alves, Jr. et al., JINST 8 (2013) P02022.

[6] F. Archilli et al., JINST 8 (2013) P10020.

[7] M. Adinolfi et al., Eur. Phys. J. C 73 (2013) 2431.

[8] R. Aaij et al., LHCb Collab., JHEP 1210 (2012) 037.

[9] R. Aaij et al., JINST 8 (2013) P04022.

[10] R. Aaij et al., LHCb Collab., JHEP 1510 (2015) 172.

[11] R. Aaij et al., LHCb Collab., JHEP 1302 (2013) 041. 
[12] R. Aaij et al., LHCb Collab., Eur. Phys. J. C 71 (2011) 1645.

[13] R. Aaij et al., LHCb Collab., JHEP 1306 (2013) 064.

[14] M. Cacciari, M. Greco and P. Nason, JHEP 9805 (1998) 007.

[15] H. S. Shao, H. Han, Y. Q. Ma, C. Meng, Y. J. Zhang and K. T. Chao, JHEP 1505 (2015) 103.

[16] M. Cacciari, M. L. Mangano and P. Nason, Eur. Phys. J. C 75, 12 (2015) 610.

[17] R. Aaij et al., LHCb Collab., JHEP 1511 (2015) 103.

[18] H. Han, Y. Q. Ma, C. Meng, H. S. Shao, Y. J. Zhang and K. T. Chao, Phys. Rev. D 94, 1 (2016) 014028.

[19] L. S. Kisslinger, M. X. Liu and P. McGaughey, Phys. Rev. D 84 (2011) 114020; Erratum: Phys. Rev. D 86 (2012) 039902.

[20] L. S. Kisslinger and D. Das, Mod. Phys. Lett. A 28 (2013) 1350120.

[21] L. S. Kisslinger and D. Das, Mod. Phys. Lett. A 29 (2014) 1450082.

[22] S. Barsuk, J. He, E. Kou and B. Viaud, Phys. Rev. D 86 (2012) 034011.

[23] R. Aaij et al., LHCb Collab., Eur. Phys. J. C 75, 7 (2015) 311.

[24] F. Maltoni and A. D. Polosa, Phys. Rev. D 70 (2004) 054014.

[25] A. Petrelli, M. Cacciari, M. Greco, F. Maltoni and M. L. Mangano, Nucl. Phys. B 514 (1998) 245.

[26] J. H. Kuhn and E. Mirkes, Phys. Rev. D 48 (1993) 179.

[27] M. Butenschoen and B. A. Kniehl, Phys. Rev. Lett. 108 (2012) 172002.

[28] K. T. Chao, Y. Q. Ma, H. S. Shao, K. Wang and Y. J. Zhang, Phys. Rev. Lett. 108 (2012) 242004.

[29] B. Gong, L. P. Wan, J. X. Wang and H. F. Zhang, Phys. Rev. Lett. 110, 4 (2013) 042002.

[30] G. T. Bodwin, H. S. Chung, U. R. Kim and J. Lee, Phys. Rev. Lett. 113, 2 (2014) 022001.

[31] M. Butenschoen, Z. G. He and B. A. Kniehl, Phys. Rev. Lett. 114, 9 (2015) 092004.

[32] R. Aaij et al., LHCb Collab., JHEP 1603 (2016) 159.

[33] R. Gauld, J. Rojo, L. Rottoli and J. Talbert, JHEP 1511 (2015) 009.

[34] B. A. Kniehl, G. Kramer, I. Schienbein and H. Spiesberger, Eur. Phys. J. C 72 (2012) 2082.

[35] L. Lyons, D. Gibaut and P. Clifford, Nucl. Instrum. Meth. A 270 (1988) 110.

[36] R. Aaij et al., LHCb Collab., Chin. Phys. C 40 (2016) no.1, 011001.

[37] M. Cacciari, S. Frixione and P. Nason, JHEP 0103 (2001) 006.

[38] M. Cacciari, S. Frixione, N. Houdeau, M. L. Mangano, P. Nason and G. Ridolfi, JHEP 1210 (2012) 137.

[39] J. L. Rosner, Phys. Rev. D 90, 1 (2014) 014023.

[40] R. Aaij et al., LHCb Collab., Phys. Rev. Lett. 114 (2015) 132001.

[41] C. H. Chang, J. X. Wang and X. G. Wu, Comput. Phys. Commun. 174 (2006) 241.

[42] M. Pivk and F. R. Le Diberder, Nucl. Instrum. Meth. A 555 (2005) 356.

[43] R. Aaij et al., LHCb Collab., Phys. Lett. B 707 (2012) 52. 
[44] R. Aaij et al., LHCb Collab., JHEP 1206 (2012) 141; Addendum: JHEP 1403 (2014) 108.

[45] R. Aaij et al., LHCb Collab., Phys. Rev. Lett. 114 (2015) 132001.

[46] A. V. Berezhnoy and A. K. Likhoded, Int. J. Mod. Phys. A 30, 20 (2015) 1550125.

[47] R. Aaij et al., LHCb Collab., JHEP 1607 (2016) 052.

[48] R. Aaij et al., LHCb Collab., JHEP 1509 (2015) 084.

[49] S. P. Jones, A. D. Martin, M. G. Ryskin and T. Teubner, JHEP 1311 (2013) 085.

[50] J. Breitweg et al., ZEUS Collab., Phys. Lett. B 437 (1998) 432.

[51] C. Adloff et al., H1 Collab., Phys. Lett. B 483 (2000) 23.

[52] S. Chekanov et al., ZEUS Collab., Phys. Lett. B 680 (2009) 4.

[53] V. P. Gonćalves, B. D. Moreira and F. S. Navarra, Phys. Lett. B 742 (2015) 172.

[54] R. Aaij et al., LHCb Collab., JHEP 1402 (2014) 072.

[55] R. Aaij et al., LHCb Collab., JHEP 1603 (2016) 133.

[56] R. Aaij et al., LHCb Collab., JHEP 1407 (2014) 094.

[57] E. G. Ferreiro, F. Fleuret, J. P. Lansberg and A. Rakotozafindrabe, Phys. Rev. C 88, 4 (2013) 047901.

[58] Z. Conesa del Valle, E. G. Ferreiro, F. Fleuret, J. P. Lansberg and A. Rakotozafindrabe, Nucl. Phys. A 926 (2014) 236.

[59] J. L. Albacete et al., Int. J. Mod. Phys. E 22 (2013) 1330007.

[60] F. Arleo and S. Peigne, JHEP 1303 (2013) 122. 